\title{
RADIOGRAPHIC DENSITY FOLLOWING GRAFTING WITH AUTOGENOUS TOOTH VERSUS AUTOGENOUS DECALCIFIED DENTIN IN SOCKET PRESERVATION: A RANDOMIZED PILOT TRIAL
}

\author{
Ahmed Hussein*, Karim Elsayid* and Samar Elkholy*
}

\begin{abstract}
Background: The extracted teeth have proven to be a ready source of bone substitute material and thus, they are no longer viewed as clinical leftover. Many techniques are employed to process the teeth into particles with different sizes, shapes and calcium composition.
\end{abstract}

Aim: The aim of the trial is to compare autogenous tooth (AT) biomaterial and the autogenous decalcified dentin (ADD) biomaterial for their efficacy in socket preservation procedures in relation to the radiographic dentistry measured on cone beam computed tomography (CBCT) images.

Methodology: A total of 12 hopeless teeth were randomly assigned into both study groups. After executing atraumatic extraction, the tooth was cleaned and milled then it was prepared into either AT or ADD with decalcification in hydrochloric acid with $\mathrm{pH}=1$ then inserted in the extraction socket. Baseline $\mathrm{CBCT}$ and the final images taken after six months were compared for radiographic density expressed in Hounsfield units $(\mathrm{Hu})$.

Results: After six months, all sites achieved soft tissue closure and were filled with bone without incidents. Radiographic bone density values were $830.67 \pm 115.5$ and $742.83 \pm 148.84$ Hounsfield units for the AT group and the ADD group respectively without a statistical difference.

Conclusions: Both types of biomaterials were similarly effective in bone healing after extraction by providing radiographic density in treated sites.

KEYWORDS: Extraction, socket preservation, bone density, biomaterial, CBCT

\section{INTRODUCTION}

Extraction of hopeless or non-restorable teeth for various reasons is still one of the frequent procedures carried out in the dental practice, but this comes with adverse consequences. Exodontia affects the individual's ability to speak, masticate and the overall quality of life and reduces the dimensions of available bone (Marcus et al., 1996). Socket preservation procedure is one of

\footnotetext{
* Department of Oral Medicine and Periodontology, Faculty of Dentistry, Cairo University, Egypt
} 
the successful attempts to reduce ridge resorption following tooth extraction. Several graft types have been used such as autogenous, allograft, xenograft or alloplastic materials (Misch and Dietsh, 1993). The use of these materials help to provide mechanical maintenance to blood clot formed inside the socket, increases the formation of bone through their osteoconductive abilities with some forms of grafts having osteoinductive properties as well (De Risi et al., 2015).

The extracted tooth is no longer viewed as clinical leftover; it has been explored as an appropriate source for autogenous graft substitute. This idea emerged due to the shared embryonic origin of bone and teeth as well as the similarities in chemical structure as dentin is composed of 30-35\% organic part and $65-70 \%$ inorganic part in comparison to alveolar bone, with inorganic and organic parts of $35 \%$ and $65 \%$ respectively (Kim et al., 2013). Decalcification is employed as it reduces the calcium content for easier degradation and replacement, widens the dentinal tubules and helps liberating several growth factors in the matrix. However, the decalcification step is laborious and may cause early discharge and dilution of growth factors into the acidic medium consequently negating their benefits (Pietrzak et al., 2011, Lee et al., 2014).

To overcome these shortcomings, the nondecalcified form has been recently employed in clinical use. Abandoning the decalcification step simplifies the processing and reducing its time and the higher calcium content may be preferable for long term stability of the graft effect (Binderman et al., 2014). In a recent systematic review, the heterogeneity in processing the teeth for graft biomaterial preparation was seen as a limitation for their use, the superiority of one graft over the other is not clear, recommending further studies for the conclusion of the best processing method in clinical applications (Gharpure and Bhatavadekar, 2018). Hence, the present study aimed to compare the effect of decalcification on both types for their radiographic effect.

\section{MATERIALS AND METHODS}

\section{Study Participants}

This study was designed as randomized two groups with 1:1 allocation, prospective pilot trial to test the difference between AT and ADD biomaterials radiographically. Participants were selected from the outpatient clinic at the Faculty of Dentistry, Cairo University, Egypt. To be eligible for the study, the following inclusion and exclusion criteria were followed:

\section{Inclusion criteria:}

- Participants having at least one single-rooted tooth indicated for extraction.

- Participants more than 18 years.

- Sockets all around the tooth indicated for extraction with a height of more than $5 \mathrm{~mm}$ for all walls.

- Motivated participants willing to complete the follow-up period.

\section{Exclusion criteria:}

- Smokers.

- Patients reporting systemic illness that may hinder healing or contraindicate surgery

- Teeth with endodontic treatment.

- Infection locally at the site of extraction.

\section{Randomization}

Twelve Participants who fulfilled the inclusion criteria and provided informed consent were randomly assigned to either test or control group by means of simple randomization with a 1:1 allocation ratio. Sequence generation was carried out through (www.randomizer.org). Numbers were randomly allocated to the two groups of the 
study. The allocation was concealed in sequentially numbered opaque sealed envelopes. Participants in both groups were equally prepared and operated and after tooth extraction and milling, the allocation sequence was revealed.

\section{Surgical intervention}

Theextraction process started withlocal anesthesia which was achieved through the administration of $2 \%$ mepivacaine $\mathrm{HCl}$ with levonordefrin 1:20000 (Alexandria Pharma Co,Egypt). Flapless atraumatic extraction started by supracrestal fiberotomy, using blade no: 15 , then periotomes 1 and 2 (Nordent Inc., USA) were inserted along the root surface; apical pressure and rocking motion were applied circumferentially to cut the periodontal ligaments. After initial luxation, forceps were used to deliver the tooth out of the socket. Following extraction, an inspection of socket integrity was carried out to ensure no granulation tissues and for evaluation of alveolar bone morphology with a periodontal probe. The extracted tooth were cleaned from cementum, periodontal ligaments, tissue attachment, caries or filling (if present) using a high-speed rotary cutting bur with saline irrigation. Following that, the tooth was ground in a manual mill into particles. To prepare the decalcified graft, particles were immersed in hydrochloric acid with a $\mathrm{pH}=1$ for at least 30 minutes in a glass container then washed two times in saline and dehydrated.

Following graft biomaterial insertion into the extraction socket, an absorbable collagen membrane was used for socket and graft biomaterial coverage. After placing the sequential graft biomaterial, the membrane was adapted over the socket orifice and an internal suture knot using 5-0 polypropylene material was placed over. Participants were prescribed Amoxicillin $500 \mathrm{mg}$ three times a day for 5 days, and Ibuprofen in case of unbearable pain. Chlorhexidine $0.12 \%$ mouthwash was prescribed as a mouthwash twice daily for two weeks.

\section{Outcome}

Radiographic analysis for bone changes was done by comparing baseline CBCT scans with six months postoperative scans. Data were exported in DICOM format and transferred to be viewed by Blue Sky Plan (MDI Europa Company, Germany). To assess radiographic density, Hounsfield units (Hu) were measured in 3 different points below the bone crest along the central line of the socket in the sagittal view on the final scans and the average was obtained. Hu values were expressed numerically.

\section{Statistical analysis}

Data were analyzed using SPSS advanced statistics (Statistical Package for Social Sciences version 26, BM Inc., Chicago, IL). Numerical data were described as mean and standard deviation. Data were explored for normality using KolmogorovSmirnov test and Shapiro-Wilk test. In the case of normally distributed numerical variables, a comparison between both treatment groups was set to be done using independent t-test. All tests were two-tailed and P-value less than or equal to 0.05 was be considered statistically significant.

\section{RESULTS}

The present study included 12 extraction sites randomly allocated into 2 groups, the intervention group utilizing the tooth graft and the control group utilizing the decalcified dentin graft. The extraction of teeth was carried according to the provisional diagnosis of being non-restorable due to deep caries or fracture and according to the patients' desire. All sites were randomly allocated into the intervention group using AT biomaterial or the control group using ADD biomaterial. All sites healed with no complications, and sockets were covered with epithelium at the end of the follow-up. Mean age of the intervention group was $37.50 \pm 8.34$ years in comparison to $32.15 \pm 5.48$ years for the control group. Both group included one male and five female 
participants. For the site of the non-restorable teeth, the intervention group had five maxillary teeth and one mandibular tooth, while the control group had three maxillary and three mandibular teeth. All differences between both groups were statistically insignificant. For the values concerning the radiographic bone density expressed in Hounsfield units, they corresponded to $830.67 \pm 115.5$ and $742.83 \pm 148.84$ for the intervention and control groups respectively without a statistical significance (table 1).

\begin{tabular}{|c|c|c|c|}
\hline & $\begin{array}{c}\text { Intervention } \\
\text { group }\end{array}$ & $\begin{array}{c}\text { Control } \\
\text { group }\end{array}$ & p-value \\
\hline Age (year) & $37.50 \pm 8.443$ & $32.15 \pm 5.48$ & 0.49 \\
\hline $\begin{array}{c}\text { Gender (male/ } \\
\text { female) }\end{array}$ & $1 / 5$ & $1 / 5$ & 1.00 \\
\hline $\begin{array}{c}\text { Site (maxilla/ } \\
\text { mandible) }\end{array}$ & $5 / 1$ & $3 / 3$ & 0.26 \\
\hline $\begin{array}{c}\text { Radiographic } \\
\text { density (Hu) }\end{array}$ & $\begin{array}{c}830.67 \pm \\
115.5\end{array}$ & $\begin{array}{c}742.83 \pm \\
148.84\end{array}$ & 0.28 \\
\hline
\end{tabular}

\section{DISCUSSION}

Socket preservation has proven to show high level success in minimizing bone loss that naturally takes place following tooth extraction when compared to negative controls. As a result, the procedure can provide for better ridge dimensions for implant placement or prosthetic rehabilitation and reduce the need for bone grafting or augmentation in the future (Weng et al., 2011, Willenbacher et al., 2016). For graft materials used in the oral cavity, there is a plethora of literature providing evidence concerning the use of allografts, xenografts or alloplasts; each with its own benefits and limitations (Kumar et al., 2013).

The bone graft abstained from teeth is a type of bone substitute that has been investigated in clinical use for sinus floor elevation, infrabony defects, bone augmentation and socket preservation. Hence, hopeless teeth are not regarded as clinical waste and can provide for an economic graft that poses growth factors and does not pose any risk of disease transmission or immunogenicity. However, literature shows heterogeneity of processing protocols for the preparation of the extracted teeth (Gharpure and Bhatavadekar, 2018). The debate about the effect of decalcification on allografts has been a hot topic for discussion in dental literature (Wood and Mealey, 2012). However, the recent review of Gharpure and Bhatavadekar in (2018) noticed that the heterogeneity in processing teeth is a limitation for their application clinically and thus, this study aimed to test the effect of decalcification on the preparation of graft material.

To minimize confounders, different measures for the selection of participants have been taken in the study. Smokers were excluded due to the fact that smoking has unfavorable effects on bone healing, not only it adversely affects host cells function and causes alternations to the inflammatory response, but also it reduces the blood supply which leads to decrease in tissue perfusion and ischemia and in turn, negatively affects healing processes following tooth extraction (Ozkan et al., 2014). Clinically, smoking can increase the post-extraction bone crest loss by $0.5 \mathrm{~mm}$ (Van der Weijden et al., 2009). Pregnant females were excluded to avoid the teratogenic effect of high radiation exposure when performing CBCT scans (Kelaranta et al., 2016).

CBCT is a highly accurate and valuable tool in dentistry; the ability to produce a three-dimensional image as well as obtaining linear measurements in axial, coronal or sagittal views has been utilized in the maxillofacial region for various purposes. CBCT is embraced due to the fact that it requires much less radiation dose compared to serial conventional tomography cuts and at the same time, provides better resolution and less distortion. (Venkatesh and Elluru, 2017, Nasseh and Al-Rawi, 2018). CBCT was chosen to measure the primary outcome as it has shown its accuracy and reproducibility as voxel size accurately corresponds to the scanned physical 
dimensions, (Sherrard et al., 2010, Shiratori et al., 2012).

In the present study, radiographic density measured on the final CBCT images expressed in Hounsfield units $(\mathrm{Hu})$ showed mean values of $830.67 \pm 115.5 \mathrm{Hu}$ for the AT group and $742.83 \pm$ $148.84 \mathrm{Hu}$ for the ADD group but the difference between both groups was not statistically significant. The radiographic density was included as the primary outcome as it can indirectly be correlated to anatomical and histological bone quality which is advantageous for successful dental implant (Silva et al., 2012, Huang et al., 2014, Almasoud et al., 2016). The value of $\mathrm{Hu}$ at the ADD group in our study came in accordance with the results of the study by Jun et al. (2014) who reported a comparable value of $981.80 \mathrm{Hu}$ when the material was used for sinus elevation procedures after 4 months of its application. Also for the use of AT, Del Canto-Díaz et al. (2019) used the AT for socket preservation and showed a comparable mean value of $922.68 \pm 250.82 \mathrm{Hu}$ after 6 months.

The autogenous graft from teeth was compared to $\beta$-tricalcium phosphate (TCP) for socket preservation in a split-mouth randomized trial including 15 patients (Joshi et al., 2016); the bone graft derived from teeth exhibited better dimensional preservation than the alloplasts after 4 months which was statistically significant. The graft derived from teeth was also compared to deproteinized bovine bone mineral (DBBM) xenograft for their effect in halting bone resorption and for the implant rehabilitation by Pang et al. (2017) and both materials were equally effective for preserving the ridge height and width as well as for implant stability afterward with no statistically significant difference.

A limitation to the present study is the inability to mask the investigator from the treatment protocol applied due to the difference in processing time between both biomaterial types as well as the difference in surface characteristics of the particles after processing. In addition, the manual preparation using bone mill is exhausting and time consuming. Another limitation to this pilot study is its small sample size included, and hence, further studies with larger sample size are required to confirm or clarify these results. It should be noticed however that the $\mathrm{Hu}$ value varies between different radiographic scans depending on the energy and quality of the $\mathrm{x}$-ray beam and thus comparison is better made when unifying these factors (Molteni, 2013, Kim, 2015a).

The autogenous tooth subtracted graft materials can circumvent some of the limitations facing the currently available graft substitutes used in dental practice. As an autogenous graft, it eradicates the risk of transmission of infectious illness and autoimmunogenicity but at the same time, does not necessitate a second surgical location when socket preservation or implant placement is performed simultaneously along extraction. It is a readily available source for obtaining graft requiring simple armamentarium and minimal cost (Binderman et al., 2014).

In conclusion, our results demonstrated that the decalcified form of the dentin yielded comparable healed bone density to the non-decalcified tooth without a significant difference. The bone substitute material obtained from both types of grafts has proved to equally provide for bone healing with adequate bone density for future implant supported or ridge supported dental prostheses.

\section{ACKNOWLEDGEMENT}

The authors would like to thank Mr. Marco Gietema for his logistic and technical support for the completion of this study. 


\section{REFERENCES}

1. Almasoud, N. N., Tanneru, N., \& Marei, H. F. (2016). Alveolar bone density and its clinical implication in the placement of dental implants and orthodontic miniimplants. Saudi medical journal, 37(6), 684-689.

2. Binderman, I., Hallel, G., Nardi, C., Yaffe, A. \& Sapoznikov, L. (2014) A Novel Procedure to Process Extracted Teeth for Immediate Grafting of Autogenous Dentin. JBR Journal of Interdisciplinary Medicine and Dental Science 02.

3. De Risi, V., Clementini, M., Vittorini, G., Mannocci, A. \& De Sanctis, M. (2015) Alveolar ridge preservation techniques: a systematic review and meta-analysis of histological and histomorphometrical data. Clinical Oral Implants Research 26, 50-68.

4. Del Canto-Díaz, A., de Elío-Oliveros, J., Del Canto-Díaz, M., Alobera-Gracia, M. A., Del Canto-Pingarrón, M. \& Martínez-González, J. M. (2019) Use of autologous toothderived graft material in the post-extraction dental socket. Pilot study. Medicina oral, patologia oral y cirugia bucal 24, e53-e60.

5. Gharpure, A. S. \& Bhatavadekar, N. B. (2018) Clinical Efficacy of Tooth-Bone Graft: A Systematic Review and Risk of Bias Analysis of Randomized Control Trials and Observational Studies. Implant Dent. 27, 119-134. Implant Dentistry 27, 119-134.

6. Huang, Y., Dessel, J. V., Depypere, M., EzEldeen, M., Iliescu, A. A., Santos, E. D., Jacobs, R. (2014). Validating cone-beam computed tomography for peri-implant bone morphometric analysis. Bone research, 2, 14010. doi:10.1038/boneres.2014.10

7. Iorio-Siciliano, V., Blasi, A., Nicolo, M., Iorio-Siciliano, A., Riccitiello, F. \& Ramaglia, L. (2017) Clinical Outcomes of Socket Preservation Using Bovine-Derived Xenograft Collagen and Collagen Membrane Post-Tooth Extraction: A 6-Month Randomized Controlled Clinical Trial. Int J Periodontics Restorative Dent 37, e290-e296.

8. Joshi, C. P., Dani, N. H. \& Khedkar, S. U. (2016) Alveolar ridge preservation using autogenous tooth graft versus beta-tricalcium phosphate alloplast: A randomized, controlled, prospective, clinical pilot study. Journal of Indian Society of Periodontology 20, 429-434.

9. Jun, S.-H., Ahn, J.-S., Lee, J.-I., Ahn, K.-J., Yun, P.-Y. \& Kim, Y.-K. (2014) A prospective study on the effectiveness of newly developed autogenous tooth bone graft material for sinus bone graft procedure. The journal of advanced prosthodontics $6,528-538$.

10. Kelaranta, A., Ekholm, M., Toroi, P. \& Kortesniemi, M. (2016) Radiation exposure to foetus and breasts from dental X-ray examinations: effect of lead shields. Dentomaxillofac Radiol 45, 20150095.

11. Kim, Y.-K., Lee, J., Um, I.-W., Kim, K.-W., Murata, M., Akazawa, T. \& Mitsugi, M. (2013) Tooth-derived bone graft material. Journal of the Korean Association of Oral and Maxillofacial Surgeons 39, 103-111.

12. Kumar, P., Vinitha, B.\& Fathima, G. (2013) Bone grafts in dentistry. J Pharm Bioallied Sci 5, S125-127.

13. Lee, E.-Y., Kim, E.-S. \& Kim, K.-W. (2014) Scanning Electron Microscopy and Energy Dispersive X-ray Spectroscopy Studies on Processed Tooth Graft Material by Vacuum-ultrasonic Acceleration. Maxillofacial Plastic and Reconstructive Surgery 36, 103-110.

14. Marcus, S. E., Drury, T. F., Brown, L. J. \& Zion, G. R. (1996) Tooth Retention and Tooth Loss in the Permanent Dentition of Adults: United States, 1988-1991. Journal of Dental Research 75, 684-695.

15. Misch, C. E. \& Dietsh, F. (1993) Bone-grafting materials in implant dentistry. Implant dentistry 2, 158-167.

16. Molteni, R. (2013) Prospects and challenges of rendering tissue density in Hounsfield units for cone beam computed tomography. Oral Surg Oral Med Oral Pathol Oral Radiol $116,105-119$.

17. Nasseh, I. \& Al-Rawi, W. (2018) Cone Beam Computed Tomography. Dent Clin North Am 62, 361-391.

18. Ozkan, A., Bayar, G. R., Altug, H. A., Sencimen, M., Dogan, N., Gunaydin, Y. \& Ergodan, E. (2014) The Effect of Cigarette Smoking on the Healing of Extraction Sockets. Journal of Craniofacial Surgery 25, e397-e402.

19. Pang, K.-M., Um, I.-W., Kim, Y.-K., Woo, J.-M., Kim, S.-M. \& Lee, J.-H. (2017) Autogenous demineralized dentin matrix from extracted tooth for the augmentation of alveolar bone defect: a prospective randomized clinical trial in comparison with anorganic bovine bone. Clinical Oral Implants Research 28, 809-815.

20. Pietrzak, W. S., Ali, S. N., Chitturi, D., Jacob, M. \& Woodell-May, J. E. (2011) BMP depletion occurs during prolonged acid demineralization of bone: characterization and implications for graft preparation. Cell and Tissue Banking 12, 81-88. 
21. Sherrard, J. F., Rossouw, P. E., Benson, B. W., Carrillo, R. \& Buschang, P. H. (2010) Accuracy and reliability of tooth and root lengths measured on cone-beam computed tomographs. Am J Orthod Dentofacial Orthop 137, S100-108.

22. Shiratori, L. N., Marotti, J., Yamanouchi, J., Chilvarquer, I., Contin, I. \& Tortamano-Neto, P. (2012) Measurement of buccal bone volume of dental implants by means of conebeam computed tomography. Clin Oral Implants Res 23, 797-804.

23. Silva, I. M., Freitas, D. Q., Ambrosano, G. M., Boscolo, F. N. \& Almeida, S. M. (2012) Bone density: comparative evaluation of Hounsfield units in multislice and cone-beam computed tomography. Braz Oral Res 26, 550-556.

24. Van der Weijden, F., Dell'Acqua, F. \& Slot, D. E. (2009) Alveolar bone dimensional changes of post-extraction sockets in humans: a systematic review. Journal of Clinical Periodontology 36, 1048-1058.
25. Venkatesh, E. \& Elluru, S. V. (2017) Cone beam computed tomography: basics and applications in dentistry. J Istanb Univ Fac Dent 51, S102-S121.

26. Weng, D., Stock, V. \& Schliephake, H. (2011) Are socket and ridge preservation techniques at the day of tooth extraction efficient in maintaining the tissues of the alveolar ridge? - Systematic review, consensus statements and recommendations of the 1st DGI Consensus Conference in September 2010, Aerzen, Germany. European Journal of Oral Implantology 4, S59-S66.

27. Willenbacher, M., Al-Nawas, B., Berres, M., Kämmerer, P. W. \& Schiegnitz, E. (2016) The Effects of Alveolar Ridge Preservation: A Meta-Analysis. Clinical Implant Dentistry and Related Research 18, 1248-1268.

28. Wood, R.A.\& Mealey, B. L. (2012) Histologic comparison of healing after tooth extraction with ridge preservation using mineralized versus demineralized freeze-dried bone allograft. J Periodontol 83, 329-336. 